\title{
Online hypergraph coloring with rejection
}

\author{
Csanád IMREH \\ University of Szeged \\ Institute of Informatics \\ email: cimreh@inf.u-szeged.hu
}

\author{
Judit NAGY-GYÖRGY \\ University of Szeged \\ Bolyai Institute \\ email: Nagy-Gyorgy@math.u-szeged.hu
}

\begin{abstract}
In this paper we investigate the online hypergraph coloring problem with rejection, where the algorithm is allowed to reject a vertex instead of coloring it but each vertex has a penalty which has to be paid if it is not colored. The goal is to minimize the sum of the number of the used colors for the accepted vertices and the total penalty paid for the rejected ones. We study the online problem which means that the algorithm receives the vertices of the hypergraph in some order $v_{1}, \ldots, v_{n}$ and it must decide about $v_{i}$ by only looking at the subhypergraph $H_{i}=\left(V_{i}, E_{i}\right)$ where $V_{i}=\left\{v_{1}, \ldots, v_{i}\right\}$ and $E_{i}$ contains the edges of the hypergraph which are subsets of $V_{i}$. We consider two models: in the full edge model only the edges where each vertex is accepted must be well-colored, in the trace model the subsets of the edges formed by the accepted vertices must be well colored as well. We consider proper and conflict free colorings. We present in each cases optimal online algorithms in the sense that they achieve asymptotically the smallest possible competitive ratio.
\end{abstract}

\section{Introduction}

A coloring of a hypergraph is an assignment of positive integers to the vertices of the hypergraph so that every edge satisfy some property. We consider

Computing Classification System 1998: F.1.2

Mathematics Subject Classification 2010: 68W27

Key words and phrases: online algorithms, hypergraph coloring, competitive ratio 
two different versions of coloring. In proper hypergraph coloring each edge must contain vertices having different colors. In conflict free (we will use the abbreviation cf) coloring each edge must contain a unique vertex which has different color to the other vertices of the edge. In the online hypergraph coloring problem the algorithm receives the vertices of the hypergraph with $n$ vertices in some order $v_{1}, \ldots, v_{n}$ and it must color $v_{i}$ by only looking at the subhypergraph $H_{i}=\left(V_{i}, E_{i}\right)$ where $V_{i}=\left\{v_{1}, \ldots, v_{i}\right\}$ and $E_{i}$ contains the edges of the hypergraph which are subsets of $V_{i}$.

We will evaluate the efficiency of the online algorithms by the competitive ratio (see $[4,10]$ ) where the online algorithm is compared to the optimal offline algorithm. We say that an online algorithm is $\mathrm{C}$-competitive if its cost is at most $\mathrm{C}$ times larger than the optimal cost.

Online proper coloring of hypergarphs first was studied in [9] where it was proven that no online algorithm exists for 2-colorable k-uniform hypergraphs which can color them with less colors than $\lceil\mathrm{n} /(\mathrm{k}-1)\rceil$, and it was proved that algorithm FF colors these hypergraphs with this much colors. This means that the best possible competitive ratio is $\lceil n /(k-1)\rceil / 2$ for this class of hypergraphs. Furthermore some special classes were also studied: the hypergraphs with given matching number and projective planes. Later randomized algorithms were studied for online proper coloring of hypergarphs in [8] where the deterministic $\Omega(n / k)$ lower bound was extended to randomized algorithms. This lower bound was also proved in the case of a more general transparent model. In [11] the online and quasionline hypergraph proper coloring problem was studied for intervals and wedges.

Online cf-coloring of hypergraph was defined in [5] where the authors considered the case where the input is a set of $n$ points on the line, and $\mathrm{R}$ is the set of the intervals of the line. They present an algorithm which uses $\mathrm{O}\left(\log ^{2}(n)\right)$ colors and also prove a matching lower bound. Online cf-coloring of intervals was further studied in [2] where several coloring models were defined and compared. The online cf-coloring of other more general hypergraphs was studied in [3] and [6].

In [7] the graph coloring problem with rejection was investigated. In this model a penalty value is assigned to each vertex and the algorithm has to choose a subset of vertices, and find a proper coloring of the induced subgraph defined by this subset. The elements of the subset are called accepted vertices the other ones are called rejected. The goal is to minimize the sum of the number of colors used to color the accepted vertices and the total penalty paid for the rejected vertices. In [7] both the online and the offline versions of the problems are investigated. 
In this paper we extend graph coloring with rejection into hypergraph coloring with rejection. There are two ways to extend the model. In the full edge model we have to color correctly only the edges where each vertices are accepted from the edge. In the trace model we have to color correctly the subhypergraph which consists of the accepted vertices and the edges which are the accepted subsets of the original edges. Note that in the special case of graphs the two models are identical. We consider both proper and cf-coloring in both models.

Main results: We studied four models since we had two possibilities for the coloring (proper and $\mathrm{cf}$ ) and two possibilities to handle rejection (full edge, trace). In the full edge model with proper coloring we present for every $\varepsilon>0$ an online algorithm $\mathcal{A}_{\varepsilon}$ and $n_{\varepsilon}$ such that $\mathcal{A}_{\varepsilon}$ is at most $\lceil\mathrm{n} /(\mathrm{k}-1)\rceil / 2+$ $\varepsilon$ competitive on $k$-uniform hypergraphs with at least $n_{\varepsilon}$ vertices for $k \geq$ 3. This competitive ratio is asymptotically the best possible since it follows from online hypergraph coloring that no online algorithm exists with smaller competitive ratio than $\lceil\mathrm{n} /(\mathrm{k}-1)\rceil / 2$ for $\mathrm{k}$-uniform hypergraphs. In case of full edge model and cf-coloring we present an $((n-1) / \varphi+\varphi)$-competitive algorithm for hypergraphs of $n$-vertices where $\varphi=(1+\sqrt{5}) / 2$. In the trace model we present an algorithm which is $(2+(n-2) / \varphi)$-competitive for both the proper and cf coloring models. All of these algorithms are asymptotically optimal since we prove that no online algorithm exists which is $(\mathrm{Cn}+\mathrm{D}$ competitive) in any these models for hypergraphs containing $n$ vertices and some constants $\mathrm{C}<1 / \varphi$, D.

\section{Notation}

In this paper on hypergraph we mean the structure $H=(V, E)$ where $V$ is the finite set of the hypergraph's vertices and $E \subseteq \rho(V)$ is the set of the edges where $\rho(V)$ is the set of the nonempty subsets of $V$. We suppose that each edge has at least two elements.

We consider the following two colorings. A proper coloring of a hypergraph is an assignment of positive integers (called colors) to the vertices of the hypergraph so that each edge contains at least two vertices with different colors. For a hypergraph $\mathrm{H}$ the minimum number of colors which is enough to color the hypergraph is called the proper chromatic number of the hypergraph and denoted by $\chi_{P}(H)$. A conflict free (cf for short) coloring of a hypergraph is an assignment of positive integers (called colors) to the vertices of the hypergraph so that each edge contains a unique color, a vertex which has different color 
to the other vertices of the edge. For a hypergraph $\mathrm{H}$ the minimum number of colors which is enough to cf-color the hypergraph is called the cf-chromatic number of the hypergraph and denoted by $\chi_{c f}(H)$.

We will consider the hypergraph coloring with rejection. This means that we can reject the coloring of some vertices, but each vertex $v$ has a penalty denoted by $p(v)$ and our goal is to minimize the sum number of used colors for the accepted vertices and the total penalty paid for the rejection of the other ones. We consider two rejection models. In the full edge model we have to color correctly only the edges where each vertices are accepted from the edge. This means that rejecting some vertex of an edge ensures that it is well colored. We also consider a different model called trace model. In this new model we consider the subhypergraph which consists of the accepted vertices and the edges which are the accepted subsets of the original edges. And this subhypergraph must to be well-colored in each step. Therefore in the trace model the rejection of some vertices of an edge does not ensure that it is well colored we have to take care of the remaining vertices. We can define the problem for both the proper and the conflict free coloring.

We consider the online problem. An online hypergraph (defined first in [1]) is a structure $\mathrm{H}_{<}=(\mathrm{H},<)$ where $\mathrm{H}$ is a hypergraph and $<$ is a linear ordering of its vertices. We call a vertex the first, second,..., and ending vertex of an edge according to the ordering $<$. An online hypergraph coloring algorithm has to color the $i$-th vertex only knowing the subhypergraph $H_{i}=\left(V_{i}, E_{i}\right)$ where $V_{i}$ contains the first $i$ vertices and $E_{i}$ contains the edges of the hypergraph which are subsets of $V_{i}$. This means that the online algorithm receives information about the edges only when the last vertex of the edge arrives. We will use the well-known greedy algorithm FF (First Fit) to color the accepted vertices of the online hypergraphs. FF uses the smallest color for each vertex which does not hurt the rule of the coloring. In case of proper coloring it uses the smallest color which does not cause a monochromatic edge. In the case of cfcoloring it uses the smallest color which does not yield an edge where none of the colors is unique. We note that in the trace model it might happen that the online algorithm is forced to accept some vertices. If it has accepted and colored two vertices by the same colors then a further vertex which forms an edge with these vertices must be accepted since otherwise the remaining edge of the subhypergraph is not well-colored. In this situation when the rejection of a vertex causes an incorrect coloring of the remaining edges we say that the vertex is forced to be accepted.

Usually in the theory of online computation the efficiency of the online algorithms is measured by the competitive ratio (see $[4,10]$ ) where the online 
algorithm is compared to the optimal offline algorithm. We denote the cost of the online algorithm $\mathcal{A}$ on an online hypergraph $\mathrm{H}_{<}$and penalty function $\mathrm{p}$ by $\mathcal{A}\left(\mathrm{H}_{<}, \mathrm{p}\right)$ and we will denote the optimal cost by opt $\left(\mathrm{H}_{<}, \mathrm{p}\right)$. An algorithm is called c-competitive if $\mathcal{A}\left(\mathrm{H}_{<}, \mathrm{p}\right) \leq \mathrm{c} \cdot \operatorname{opt}\left(\mathrm{H}_{<}, \mathrm{p}\right)$ for every $\mathrm{H}$ and $\mathrm{p}$. Since no constant competitive online algorithm exists we will consider the competitive ratio as a function of the number of vertices, denoted by $n$.

We also use the following notion from the theory of hypergraphs. A hypergraph is called $k$-uniform if each edge contains $k$ vertices.

\section{Online coloring hypergraphs with rejection in the full edge model}

\subsection{Proper coloring}

Note that a lower bound of $[n /(k-1)\rceil / 2$ on $k$-uniform 2-proper-colorable hypergraphs for $k \geq 3$ comes from the case without rejection. Surprisingly, the following theorem shows that one can reach this competitive ratio in the asymptotical sense for the more general case where rejection is also allowed.

Theorem 1 If $\mathrm{k} \geq 3$, then for every $\varepsilon<1 / 8$ there is an online algorithm $\mathcal{A}_{\varepsilon}$ and $\mathrm{n}_{\varepsilon}$, such that $\mathcal{A}_{\varepsilon}$ is at most $\lceil\mathrm{n} /(\mathrm{k}-1)\rceil / 2+\varepsilon$ competitive on $\mathrm{k}$-uniform hypergraphs with at least $\mathrm{n}_{\varepsilon}$ vertices.

Proof. Let $\delta_{\varepsilon}=2 \varepsilon /(k-2), n_{\varepsilon}=\frac{2 k-2}{\delta_{\varepsilon}-\delta_{\varepsilon}^{2}(2 k-2)}$. Define the following algorithm:

Algorithm $\mathcal{A}_{\varepsilon}:$ If the penalty of the next vertex is less then $\delta_{\varepsilon}$ reject it, otherwise color it by algorithm FF.

Denote by $A$ the set of the colored and by $B$ the set of the rejected vertices by $\mathcal{A}_{\varepsilon}$, and $\chi_{\mathcal{A}_{\varepsilon}}(\mathrm{A})$ the number of colors used by $\mathcal{A}_{\varepsilon}$. Let $n=|\mathrm{A}|+|\mathrm{B}|$. Then the cost of $\mathcal{A}_{\varepsilon}$ is $\chi_{\mathcal{A}_{\varepsilon}}(\mathrm{A})+\mathrm{p}(\mathrm{B}) \leq\lceil|\mathrm{A}| /(\mathrm{k}-1)\rceil+\delta_{\varepsilon}|\mathrm{B}|$.

We have three cases.

Case 1. Suppose that the optimal algorithm uses at least 2 colors. In this case its cost is at least 2 . Therefore we obtain that 


$$
\frac{\operatorname{cost}_{\mathcal{A}_{\varepsilon}}\left(\mathrm{H}_{<,}, \mathrm{p}\right)}{\operatorname{opt}\left(\mathrm{H}_{<}, \mathrm{p}\right)} \leq \frac{\operatorname{cost}_{\mathcal{F F}_{\mathcal{E}}}\left(\mathrm{H}_{<,} \mathrm{p}\right)}{2} \leq \frac{1}{2}\left\lceil\frac{\mathrm{n}}{\mathrm{k}-1}\right\rceil
$$

Case 2. Suppose that the optimal algorithm uses one color. We state that in this case it must reject at least $\chi_{\mathcal{A}_{\varepsilon}}(A)-1$ vertices from $A$. First observe that $\mathrm{FF}$ uses color $\boldsymbol{j}$ for a new vertex only when the vertex ends for each $i<j$ and edge containing $k-1$ vertices colored by $i$. Therefore for each pair of color classes of A there exists an edge which contains only vertices from these color classes. This means that the optimal algorithm cannot accept all vertices from two different color classes of A since it could not color them correctly by 1 color. Thus it follows that the optimal algorithms must reject at least $\chi_{\mathcal{A}_{\varepsilon}}(A)-1$ vertices from $A$.

If $|A|>0$ then

$$
\begin{aligned}
\frac{\operatorname{cost}_{\mathcal{A}_{\varepsilon}}\left(H_{<,} p\right)}{\operatorname{opt}\left(H_{<}, p\right)} & \leq \frac{\chi_{\mathcal{A}_{\varepsilon}}(A)+p(B)}{1+\delta_{\varepsilon}\left(\chi_{\mathcal{A}_{\varepsilon}}(A)-1\right)} \\
& \leq \frac{\chi_{\mathcal{A}_{\varepsilon}}(A)}{\delta_{\varepsilon}\left(\chi_{\mathcal{A}_{\varepsilon}}(A)\right)}+p(B) \\
& \leq \frac{1}{\delta_{\varepsilon}}+\delta_{\varepsilon}|B| \\
& \leq \frac{1}{\delta_{\varepsilon}}+\delta_{\varepsilon} n \\
& \leq \frac{n}{2(k-1)}
\end{aligned}
$$

where the last inequality comes from the definition of $n_{\varepsilon}$.

If $|A|=0$ then

$$
\frac{\operatorname{cost}_{\mathcal{A}_{\varepsilon}}\left(H_{<, p}\right)}{\operatorname{opt}\left(H_{<}, p\right)} \leq p(B) \leq \delta_{\varepsilon} n \leq \frac{n}{2(k-1)}
$$

Case 3. Suppose that the optimal algorithm uses no colors, i.e. it rejects all vertices. If $|A|=0$ then $\mathcal{A}_{\varepsilon}$ optimal. Otherwise 


$$
\begin{aligned}
\frac{\operatorname{cost}_{\mathcal{A}_{\varepsilon}}\left(H_{<}, p\right)}{\operatorname{opt}\left(H_{<}, p\right)} & \leq \frac{\chi_{\mathcal{A}_{\varepsilon}}(A)+p(B)}{p(A)+p(B)} \\
& \leq \frac{\chi_{\mathcal{A}_{\varepsilon}}(A)}{p(A)} \\
& \leq \frac{\frac{|A|}{k-1}+\frac{k-2}{k-1}}{\delta_{\varepsilon}|A|} \\
& \leq \frac{1}{\delta_{\varepsilon}(k-1)}+\frac{k-2}{\delta_{\varepsilon}(k-1)}=\frac{1}{\delta_{\varepsilon}} \\
& \leq \frac{n}{2(k-1)}
\end{aligned}
$$

where the first and third inequality come from the definition of the algorithm and the case, the fourth one by $|A| \geq 1$, the last two come from the definition of $n_{\varepsilon}$.

\subsection{Conflict-free coloring}

In the full edge model for cf coloring we considered the following algorithm.

Algorithm $\mathcal{B}$ : If the penalty of the next vertex is less then $1 / \varphi$ reject it, otherwise color it by FF.

Theorem 2 Algorithm $\mathcal{B}$ is $(\mathrm{n}-1) / \varphi+\varphi$-competitive on hypergraphs on $\mathrm{n}$ vertices where $\varphi=(1+\sqrt{5}) / 2$.

Proof. Consider an input hypergraph denoted by $\mathrm{H}_{<}$. Denote $A$ the set of the colored and $B$ the set of the rejected vertices by $\mathcal{B}$, and $\chi_{\mathcal{B}}(A)$ the number of colors used by $\mathcal{B}$. Let $n=|A|+|B|$. Then the cost of $\mathcal{B}$ is

$$
\chi_{\mathcal{B}}(A)+p(B) \leq|A|+|B| / \varphi .
$$

We have three cases.

Case 1. Suppose that the optimal algorithm uses at least 2 colors. In this case the optimal cost is at least 2 , therefore 


$$
\begin{aligned}
\frac{\operatorname{cost}_{\mathcal{B}}\left(H_{<}, p\right)}{\operatorname{opt}\left(H_{<,} p\right)} & \leq \frac{\chi_{\mathcal{B}}(\mathrm{A})+p(B)}{2} \leq \frac{|A|+|\mathrm{B}| / \varphi}{2} \\
& \leq \frac{n}{2} \leq \frac{n-1}{\varphi}+\varphi .
\end{aligned}
$$

Case 2. Suppose that the optimal algorithm uses one color. If the input hypergraph has edges colored by $\mathcal{B}$ then the optimal algorithm have to reject at least one vertex with penalty at least $1 / \varphi$. Therefore the optimal cost is at least $1+1 / \varphi$, thus we obtain that

$$
\begin{aligned}
\frac{\operatorname{cost}_{\mathcal{B}}\left(\mathrm{H}_{<,} p\right)}{\operatorname{opt}\left(\mathrm{H}_{<,}, p\right)} & \leq \frac{\chi_{\mathcal{B}}(\mathrm{A})+\mathrm{p}(\mathrm{B})}{1+1 / \varphi} \\
& \leq \frac{|\mathrm{A}|+|\mathrm{B}| / \varphi}{\varphi} \leq \frac{\mathrm{n}}{\varphi}
\end{aligned}
$$

If the input hypergraph has no edge colored by $\mathcal{B}$ then the optimal cost is at least 1 but in this case $\chi_{\mathcal{B}}(\mathcal{A}) \leq 1$ thus

$$
\frac{\operatorname{cost}_{\mathcal{B}}\left(H_{<, p}\right)}{\operatorname{opt}\left(H_{<}, p\right)} \leq \frac{\chi_{\mathcal{B}}(A)+p(B)}{1} \leq 1+(|B|) / \varphi \leq 1+\frac{n-1}{\varphi} .
$$

Case 3. Suppose that the optimal algorithm uses no colors, i.e. it rejects all vertices. Then

$$
\frac{\operatorname{cost}_{\mathcal{B}}\left(H_{<, p}\right)}{\operatorname{opt}\left(H_{<,} p\right)} \leq \frac{\chi_{\mathcal{B}}(A)+p(B)}{p(A)+p(B)} \leq \frac{\chi_{\mathcal{B}}(A)}{p(A)} \leq \frac{|A|}{|A| / \varphi}=\varphi \leq \frac{n-1}{\varphi}+\varphi .
$$

Note that considering the online cf-coloring without rejection of 2-cf-colorable hypergraphs we can obtain the following result.

Lemma 3 No online cf-coloring algorithm uses less then $\mathrm{n}-1$ colors on 2cf-colorable hypergraphs on $\mathrm{n}$ vertices.

Proof. Give vertices until two of them are colored by the same color. Suppose that online algorithm colors $v_{i}$ and $v_{j}$ with the same color. Then reveal edges in the $m$-th phase $\left\{v_{i}, v_{j}, v_{m}\right\}$ and $\left\{v_{i}, v_{j}, v_{\ell}, v_{m}\right\}$ for all $\ell<m, \ell \neq i, j$. Then the online algorithm must use a new color for each new vertex. 
This hypergraphs is 2-cf-colorable: $v_{i}$ is in the first color class and the other vertices are in the second.

This observation proves that no online algorithm can be better than $(n-1) / 2$ competitive for online cf-coloring of hypergraphs, and this bounds holds as well for the model with rejection since we can use penalty $\infty$ for all vertices.

On the other hand we can extend the idea of this lower bound to the model with rejection and prove that the competitive ratio of $\mathcal{B}$ is the best possible in the asymptotical sense as the following theorem shows.

Theorem 4 No online algorithm exists which is $\mathrm{Cn}+\mathrm{D}$-competitive in the problem of conflict free coloring with rejection in the full edge model for hypergraphs containing $n$ vertices for some constants $\mathrm{C}<1 / \varphi$ and $\mathrm{D}$.

Proof. Suppose that, on the contrary, there exist constants $C<1 / \varphi$ and $\mathrm{D}$ and an online algorithm $\mathcal{C}$ which is $\mathrm{C} n+\mathrm{D}$-competitive. First we present vertices with penalty $1 / \varphi$ and no edge until two of them are colored by the same color or the number of vertices reaches a number $n_{1}>D /(1 / \varphi-C)$. If none of these vertices received the same color then the sequence ends, the optimal algorithm colors these vertices by one color and its cost is 1 . The online algorithms pays at least $1 / \varphi$ for each of them thus the online cost is at least $n_{1} / \varphi$ and we obtain a contradiction since $n_{1} / \varphi>C n_{1}+D$ by the definition of $n_{1}$.

Now suppose that the online algorithm colors two accepted vertices by the same colors. Let these vertices be $v_{i}$ and $v_{k}$, where $i<k$. Note that the first phase of the inputs ends by vertex $v_{k}$. In this case we continue the sequence with the points $v_{k+1}, \ldots, v_{n}$ where $n>(D+k / \varphi) /(1 / \varphi-C)$. Each such vertex $v_{\mathrm{q}}$ has penalty $\infty$, and each of them ends the edges $\left(v_{i}, v_{k}, v_{\mathrm{q}}\right)$ and $\left(v_{i}, v_{k}, v_{s}, v_{\mathrm{q}}\right)$ for $s<\mathrm{q}$ and $s \neq i, k$. Then each such vertex must be accepted, and these edges force a new color for each of them. Therefore, $v_{i}$ and $v_{k}$ have the same color and the other accepted vertices are colored by different colors. Thus the cost of the online algorithm is $m / \varphi+n-m-1$, where $m$ is the number of rejected vertices in the first phase. Therefore its cost is at least $(k-2) / \varphi+n-k+1$. On the other hand an optimal algorithm rejects $v_{i}$ and accepts all the other vertices and colors them by color 1 . Then its cost is $1+1 / \varphi$. Therefore the ratio of the online and offline costs is at least

$$
\frac{(k-2) / \varphi+n-k+1}{1+1 / \varphi}>\frac{n}{\varphi}-\frac{k}{\varphi}>C n+D,
$$

and the theorem follows. 


\section{The trace model}

In the trace model we analyze the following online algorithm. The same algorithm is defined for both the proper and cf-colorings, the difference comes from the fact that the algorithm uses FF to color the vertices, and it might assign different colors in the two models. Moreover the set of the accepted vertices might be different in the models since it depends on the previous vertices and also on the model whether a vertex is forced to be accepted or not.

$\mathcal{D}$ : If the penalty of the next vertex is less then $1 / \varphi$ and the vertex is not forced to be accepted then reject it. Otherwise color the first accepted vertex by color 1 , the second one by color 2 and the further accepted vertices by algorithm FF.

Theorem 5 Algorithm $\mathcal{D}$ is $2+(\mathrm{n}-2) / \varphi$-competitive in both trace coloring models (proper and $\mathrm{cf}$ ), where $\mathrm{n}$ is the number of vertices.

Proof. Consider an input hypergraph by $\mathrm{H}_{<}$. Again we have three cases.

Case 1. First suppose that the optimal algorithm uses at least two colors to color its accepted subhypergraph. In this case opt $\left(\mathrm{H}_{<}, \mathrm{p}\right) \geq 2$. On the other hand $\operatorname{cost}_{\mathcal{D}}\left(\mathrm{H}_{<}, \mathrm{p}\right) \leq \mathrm{n}$ is obviously valid since the algorithm pays less than 1 penalty for the rejected vertices and uses at most one color for the accepted ones. Therefore in this case the theorem follows by $\varphi<2$.

Case 2. Now suppose that the optimal algorithm uses one color to color its accepted subhypergraph. Denote the set of its rejected vertices by $R_{O P T}$. Then the optimal cost is $1+p\left(R_{\text {OPT }}\right)$.

If $\mathcal{D}$ rejects all of the vertices from $R_{\mathrm{OPT}}$ then its accepted vertices are colored by at most 2 colors. Thus the cost of the algorithm is at most $2+(n-2) / \varphi$ and the results follows since the optimal cost is at least 1.

Suppose $R_{\text {OPT }}$ contains some vertex which is accepted by $\mathcal{D}$. The first such vertex is not forced to be accepted by $\mathcal{D}$, since otherwise the optimal algorithm could not color its accepted vertices by one color. Thus its penalty is at least $1 / \varphi$. This yields that $\operatorname{opt}\left(\mathrm{H}_{<}, \mathrm{p}\right) \geq 1+1 / \varphi$ and by $\operatorname{cost}_{\mathcal{D}}\left(\mathrm{H}_{<}, \mathrm{p}\right) \leq \mathrm{n}$ we obtain that

$$
\frac{\operatorname{cost}_{\mathcal{D}}\left(\mathrm{H}_{<}, \mathrm{p}\right)}{\operatorname{opt}\left(\mathrm{H}_{<,}, \mathrm{p}\right)} \leq \frac{\mathrm{n}}{1+1 / \varphi}=\frac{\mathrm{n}}{\varphi} \leq 2+(\mathrm{n}-2) / \varphi .
$$

Case 3. Finally, suppose that the optimal algorithm uses 0 color which means that it rejects all vertices. Then its cost is the sum of the penalties of the vertices. No consider the following two subcases.

First suppose that some forced vertex is colored by $\mathcal{D}$. To have a forced vertex it needs at least two accepted vertices with the same color which means 
that it has accepted at least 3 unforced vertices. On the other hand each of these vertices has penalty at least $1 / \varphi$. This yields that the total penalty of the vertices thus the optimal cost is at least $3 / \varphi>1+1 / \varphi$ and we obtain again that

$$
\frac{\operatorname{cost}_{\mathcal{B}}\left(\mathrm{H}_{<,} \mathrm{p}\right)}{\operatorname{opt}\left(\mathrm{H}_{<}, \mathrm{p}\right)} \leq \frac{\mathrm{n}}{1+1 / \varphi}=\frac{\mathrm{n}}{\varphi} \leq 2+(\mathrm{n}-2) / \varphi .
$$

Finally suppose that the optimal algorithm uses 0 color and there exists no forced vertex accepted by $\mathcal{D}$. Then let $A$ be the set of the vertices accepted by $\mathcal{D}$ and $\mathrm{B}$ be the set of vertices rejected by $\mathcal{D}$. We obtain that

$$
\frac{\operatorname{cost}_{\mathcal{B}}\left(H_{<,} p\right)}{\operatorname{opt}\left(H_{<}, p\right)} \leq \frac{|A|+p(B)}{p(A)+p(B)} \leq \frac{|A|}{|A| / \varphi}=\varphi \leq 2+\frac{n-2}{\varphi} .
$$

Now we prove that the bound is tight in the sense that no asymptotically better online algorithm exists. We use a similar construction as we did in the case of cf coloring in the full edge model. The lower bound is again true for both the proper and cf colorings.

Theorem 6 No online algorithm exists which is $\mathrm{Cn}+\mathrm{D}$-competitive in the trace model for proper or cf-coloring with rejection for hypergraphs containing $\mathrm{n}$ vertices and some constants $\mathrm{C}<1 / \varphi$ and $\mathrm{D}$.

Proof. Suppose that we have an online algorithm which has better competitive ratio, denote it by $\mathcal{E}$. First we present vertices with penalty $1 / \varphi$ and no edge until two of them are not colored by the same color or the number of vertices reaches $n_{1}>D /(1 / \varphi-C)$. If none of these vertices received the same color then the sequence ends the optimal algorithm colors these vertices by one color and its cost is 1 . The online algorithms pays at least $1 / \varphi$ for each of them thus the online cost is at least $n_{1} / \varphi$ and we obtain a contradiction.

Now suppose that the online algorithm colors two accepted vertices by the same colors. Let these vertices be $v_{i}$ and $v_{k}$ where $i<k$. Note that the first phase of the inputs ends by vertex $v_{k}$. In this case we continue the sequence with the points $v_{k+1}, \ldots, v_{n}$ where $n>(D+k / \varphi) /(1 / \varphi-C)$. Each such vertex $v_{\mathrm{q}}$ has penalty 0 , and each of them ends the edges $\left(v_{i}, v_{k}, v_{q}\right)$ and $\left(v_{p}, v_{q}\right)$ for $p=1, \ldots, q-1$. Then the first edge forces the acceptance of the vertex (otherwise the remaining edge $\left(v_{i}, v_{k}\right)$ would not be well-colored). Moreover the other edges ensures that each vertex must receive a new color. Therefore, $v_{i}$ and $v_{k}$ have the same color and the other accepted vertices are colored by 
different colors. Thus the cost of the online algorithm is $m / \varphi+n-m-1$, where $m$ is the number of rejected vertices in the first phase. This yields that its cost is at least $(k-2) / \varphi+n-k+1$. On the other hand an optimal algorithm accepts the vertices $v_{1}, \ldots, v_{k-1}$ colors them by color 1 and rejects the further vertices. Then its cost is $1+1 / \varphi$. Therefore the ratio of the online and offline costs is at least

$$
\frac{(k-2) / \varphi+n-k+1}{1+1 / \varphi}>\frac{n}{\varphi}-\frac{k}{\varphi}>C n+D
$$

and the theorem follows.

\section{Acknowledgements}

This work was supported by the European Union and the European Social Fund through project Telemedicina (Grant no.: TÁMOP-4.2.2.A-11/1/KONV2012-0073 ). Cs. Imreh was supported by the return fellowship of the Alexander von Humboldt Foundation. J. Nagy-György was supported by the European Union and the State of Hungary, co-financed by the European Social Fund in the framework of TÁMOP 4.2.4. A/2-11-1-2012-0001 National Excellence Program.

\section{References}

[1] N. Alon, U. Arad, Y.Azar, Independent Sets in Hypergraphs with Applications to Routing Via Fixed Paths, Proc. 2nd International Workshop on Approximation Algorithms for Combinatorial Optimization Problems (APPROX99), Lecture Notes in Computer Science 1671 (1999) 16-27. $\Rightarrow 8$

[2] A. Bar-Noy, P. Cheilaris and S. Smorodinsky, Conflict-free coloring for intervals: from offline to online, Proc. 18th Annual ACM Parallelism Algorithms Architectures (SPAA06), 2006, pp. 128-137. $\Rightarrow 6$

[3] A. Bar-Noy, P. Cheilaris, S. Olonetsky, and S. Smorodinsky, Online conflict-free colorings for hypergraphs, Proc. 34th International Colloquium on Automata, Languages and Programming (ICALP07), Lecture Notes in Computer Science 4596 (2007) 219-230. $\Rightarrow 6$

[4] A. Borodin, R. El-Yaniv, Online Computation and Competitive Analysis, Cambridge University Press, 1998. $\Rightarrow 6,8$

[5] K. Chen, A. Fiat, H. Kaplan, M. Levy, J. Matousek, E. Mossel, J. Pach, M. Sharir, S. Smorodinsky, U. Wagner and E. Welzl, Online conflict-free coloring for intervals, SIAM J. Computing 36 (2006) 1342-1359. $\Rightarrow 6$ 
[6] K. Chen, H. Kaplan, M. Sharir, Online CF coloring for halfplanes, congruent disks, and axis-parallel rectangles, ACM Trans. Algorithms 5 (2009) Article No. 16 . $\Rightarrow 6$

[7] L. Epstein, A. Levin, G. J. Woeginger, Graph coloring with rejection, J. Comput. System Sci., 77, 2 (2011) 439-447. $\Rightarrow 6$

[8] M. M. Halldorsson, Online coloring of hypergraphs, Inform. Process. Lett. 110, 10 (2010) 370-372. $\Rightarrow 6$

[9] Cs. Imreh, J. Nagy-György, Online hypergraph coloring, Inform. Process. Lett. 109, $4(2008) 23-26 . \Rightarrow 6$

[10] Cs. Imreh, Competitive analysis, in: Algorithms of Informatics, Vol. 1. Foundations (ed. A. Iványi), mondAt Kiadó, Budapest, 2007, pp. 395-428. $\Rightarrow 6$, 8

[11] B. Keszegh, N. Lemons, D. Pálvölgyi, Online and quasi-online colorings of wedges and intervals, Proc. 39th International Conference on Current Trends in Theory and Practice of Computer Science (SOFSEM 2013), Lecture Notes in Computer Science $\mathbf{7 7 4 1}$ (2013) 292-306. $\Rightarrow 6$

Received: August 11, 2014•Revised: April 7, 2015 